\title{
OPEN The role of lipoprotein profile in depression and cognitive performance: a network analysis
}

\author{
Qiu-fang Jia ${ }^{1,6}$, Han-xue Yang ${ }^{2,6}$, Nan-nan Zhuang ${ }^{1,6}$, Xu-yuan Yin $^{1}$, Zhen-hua Zhu ${ }^{1}$, \\ Ying Yuan ${ }^{1}$, Xiao-li Yin ${ }^{3}$, Yi Wang ${ }^{2}$, Eric F. C. Cheung ${ }^{4}$, Raymond C. K. Chan ${ }^{2,5 \bowtie}$ \& Li Hui ${ }^{1 \bowtie}$
}

Lipid profile (total cholesterol and lipoprotein fractions) has been found to correlate with depression and cognitive impairment across the lifespan. However, the role of lipid levels in self-rated depressive state and cognitive impairment remains unclear. In this study, we examined the relationship between lipid profile (total cholesterol, triglycerides, high-density lipoprotein cholesterol and low-density lipoprotein cholesterol) and cognition in adults with and without self-rated depression. Four hundred and thirty-eight healthy participants completed the Repeatable Battery for the Assessment of Neuropsychological Status (RBANS), the Self-Rating Depression Scale (SDS), and a serum lipoprotein test. Using multivariate ANOVA, partial correlation and network analysis, a network linking lipoprotein profile, depressive state and cognition was constructed. A significant difference in serum lipid profile between the high and low depressive groups was detected. Depressive state had a strong negative correlation with cognitive performance. Of the lipid profile, only high-density lipoprotein was positively correlated with depressive symptom severity, whereas the other three indices showed negative correlation with both depressive state and cognitive performance. Our results suggest that serum lipid profile may be directly linked to self-rated depression and cognitive performance. Further studies recruiting larger clinical samples are needed to elucidate the specific effect of lipoprotein on cognitive impairment in mood disorder.

Depression is characterized by significant cognitive impairment ${ }^{1,2}$. Previous research has shown that serum lipid levels are associated with cognitive function, although some of the results are mixed. Evidence suggests that high-density lipoprotein (HDL) may be able to boost cognitive function ${ }^{3-6}$, with a more pronounced effect on memory among women ${ }^{7}$. As for total cholesterol (CHO), low-density lipoprotein (LDL) and triglycerides (TG), some have observed that higher $\mathrm{CHO}^{8}$, higher $\mathrm{LDL}^{7,9}$ and lower $\mathrm{TG}^{8,10}$ are associated with better function in most cognitive domains, whereas other studies have found no significant correlation between these three lipoproteins and cognitive function ${ }^{11,12}$, or an opposite effect ${ }^{8,13}$. Also, a detrimental role of $\mathrm{CHO}$ on memory and hippocampal morphology has been reported in an animal study ${ }^{14}$. Notably, a direct pathway from serum lipid levels to cognitive impairment in depression has also been reported ${ }^{15}$, but the above four serum lipid indices have been suggested to have somewhat different roles. For instance, a Chinese population-based study concluded that only higher TG level contributed to greater cognitive impairment in major depressive disorder, especially in delayed memory ${ }^{15}$.

The role of serum lipid levels in depression has also been established in elderly men ${ }^{16}$, middle-aged men ${ }^{17}$, young adult women ${ }^{18}$, and general population across the lifespan ${ }^{19}$. Yet conclusions regarding the correlation between serum lipid profile and depressive symptoms are inconsistent, ranging from direct to inverse and sometimes no correlation ${ }^{20}$. An inverse relationship between depressive symptoms and higher $\mathrm{CHO}$ level $^{21-23}$ and higher LDL level ${ }^{18,24}$ have been reported. One study reported that low HDL and high LDL levels may be correlated with more severe depressive symptoms in a Chinese sample ${ }^{25}$, while another study has found that depression may be correlated with low LDL level rather than $\mathrm{HDL}_{\text {level }}{ }^{26}$. Drawing on their own results, Igna and colleagues proposed an "unfavourable serum lipid profile" characterized by higher HDL level, lower LDL and CHO levels ${ }^{24}$.

\footnotetext{
${ }^{1}$ The Affiliated Guangji Hospital of Soochow University, Medical College of Soochow University, Suzhou, Jiangsu, People's Republic of China. ${ }^{2}$ Neuropsychology and Applied Cognitive Neuroscience Laboratory, CAS Key Laboratory of Mental Health Institute of Psychology, Beijing, People's Republic of China. ${ }^{3}$ Wenzhou Kangning Hospital, Wenzhou Medical University, Wenzhou, Zhejiang, People's Republic of China. ${ }^{4}$ Castle Peak Hospital, Hong Kong, People's Republic of China. ${ }^{5}$ Department of Psychology, University of Chinese Academy of Sciences, Beijing, People's Republic of China. ${ }^{6}$ These authors contributed equally: Qiu-fang Jia, Han-xue Yang and Nan-nan Zhuang. ${ }^{\square}$ email: rckchan@psych.ac.cn; huili004100@126.com
} 


\begin{tabular}{|l|c|}
\hline Characteristics & Total sample (N=438) \\
\hline Age (years), mean (SD) & $34.83(1.09)$ \\
\hline Gender, female\% (n) & $71.2(312)$ \\
\hline Education (years), mean (SD) & $12.74(3.29)$ \\
\hline BMI, mean (SD) & $22.09(2.89)$ \\
\hline Smoking, \% (n) & $84.5(370)$ \\
\hline No & $2.3(10)$ \\
\hline Former & $13.2(58)$ \\
\hline Current & $4.86(1.22)$ \\
\hline CHO, mmol/L, median (IQR) & $1.47(0.42)$ \\
\hline HDL, mmol/L, median (IQR) & $2.75(1.05)$ \\
\hline LDL, mmol/L, median (IQR) & $1.37(0.85)$ \\
\hline TG, mmol/L, median (IQR) & $85.71(13.13)$ \\
\hline RBANS total score, mean (SD) & $83.25(16.24)$ \\
\hline Immediate Memory & $77.80(13.41)$ \\
\hline Visual-spatial/Constructional span & $92.41(15.17)$ \\
\hline Language & $105.26(17.91)$ \\
\hline Attention & $87.42(11.28)$ \\
\hline Delayed memory & $38.83(8.66)$ \\
\hline SDS standardized total score, mean (SD) &
\end{tabular}

Table 1. Characteristics of participants. BMI body mass index, $C H O$ total cholesterol, $H D L$ high-density lipoprotein cholesterol, $L D L$ low-density lipoprotein cholesterol, $T G$ Triglycerides, RBANS Repeatable Battery for the Assessment of Neuropsychological Status, SDS Self-rating Depression Scale, SD standard deviation, IQR inter-quartile ranges.

In addition, they suggested that age, education level, smoking habit and body mass index (BMI) are key mediating factors that have significant negative impact on decreased HDL and increased LDL levels ${ }^{18,24}$. Pan et al. ${ }^{27}$ also found significant correlations between individual components of the metabolic syndrome (CHO and TG) and depression in their meta-analysis on the bidirectional association between depression and metabolic syndrome. More recently, a meta-analytic review has linked depression with overall lower serum LDL level, though high heterogeneity was also noted ${ }^{28}$.

The aim of this study was to examine the relationship between lipoprotein profile (CHO, TG, HDL, and LDL) and cognitive function in adults with and without self-rated depression. Most previous studies that explored the relationship between serum lipid levels and depression used multiple linear regression analysis after adjusting for confounding factors ${ }^{29}$. Here we adopted the network analysis approach, a new visualization tool that takes into account partially controlled correlational links among conceptualized variables ${ }^{30}$. We hypothesized that: (1) in general, significant negative correlations would be present between depressive state and cognitive performance, while serum lipoprotein levels would associate positively with depressive state and negatively with cognitive performance; (2) people with high and low self-rated depressive state would differ in cognitive function and serum lipoprotein levels; and (3) major demographic and lifestyle confounding factors (i.e. age, sex, education level, smoking status and BMI) would have a significant effect on serum lipoprotein levels, cognitive function, depression status and their relationships.

\section{Results}

Sample characteristics and group differences. Table 1 summarizes the demographic, lipoprotein and cognitive information of the sample. The Self-Rating Depression Scale (SDS) total score did not differ between male and female participants after controlling for age. There was no significant difference in sex, education level, smoking status, BMI, and lipoprotein levels between the low-depressive (LD) and high-depressive (HD) groups. Although we sought to recruit individuals with and without depressive symptom in the study, all participants with a history of any physical and mental illness including major depressive disorder, alcohol and drug dependence were also excluded. Therefore, only a small part of the participants was classified into the HD group. The HD group was significantly older than the LD group $(\mathrm{F}=2.17, p=0.03)$. However, all lipoprotein levels except HDL were found to be slightly higher in the LD group compared with the HD group. This pattern was reaffirmed in the subsequent multivariate ANOVA after controlling for confounding factors, in which significant discrepancies in $\mathrm{CHO}(\mathrm{F}=3.821, \mathrm{p}=0.002)$, TG $(\mathrm{F}=15.649, p<0.001)$, LDL $(\mathrm{F}=3.654,0.003)$ and $\operatorname{HDL}(\mathrm{F}=7.606, p<0.001)$ levels were detected between the two groups. As for cognitive function, the LD group showed significantly higher scores on the Repeatable Battery for the Assessment of Neuropsychological Status (RBANS) except for the "language" subscale compared with the HD group. Moreover, after controlling for confounding factors, all sub-scale scores differed significantly between the two groups (see Table 2). Additionally, in our whole sample, BMI did not show any significant correlations with either depressive state $(\mathrm{r}=0.018)$ or total RBANS score $(\mathrm{r}=-0.079)$. 


\begin{tabular}{|l|l|l|r|l|l|l|}
\hline Indexes & LD $(\mathbf{n}=\mathbf{3 7 9})$ & HD $(\mathbf{n}=\mathbf{5 9})$ & $\mathbf{F}(\mathbf{t})$ & $\boldsymbol{p}$ & Adjusted F & Adjusted $\boldsymbol{p}$ \\
\hline Age (mean, SD) & $35.27(10.77)$ & $31.98(11.04)$ & 2.17 & $0.03^{*}$ & - & - \\
\hline Gender (female\%) & 70.2 & 78.0 & -1.23 & 0.22 & - & - \\
\hline Education (years) & $12.83(3.19)$ & $12.17(3.85)$ & 1.45 & 0.15 & - & - \\
\hline BMI (mean, SD) & $22.04(3.00)$ & $22.44(3.56)$ & -0.94 & 0.35 & - & - \\
\hline Cognitive function & $83.95 \pm 16.04$ & $78.79 \pm 16.91$ & 5.279 & $0.022^{*}$ & 10.53 & $<0.001^{* *}$ \\
\hline Immediate memory & $78.51 \pm 13.47$ & $73.22 \pm 12.17$ & 7.984 & $0.005^{* *}$ & 7.47 & $<0.001^{* *}$ \\
\hline Visual-spatial/constructional & $92.79 \pm 15.03$ & $89.96 \pm 15.96$ & 1.756 & 0.186 & 8.06 & $<0.001^{* *}$ \\
\hline Language & $106.16 \pm 17.40$ & $99.47 \pm 20.13$ & 7.103 & $0.008^{* *}$ & 22.44 & $<0.001^{* *}$ \\
\hline Attention & $88.18 \pm 10.68$ & $82.58 \pm 13.65$ & 12.950 & $0.000^{* *}$ & 5.92 & $<0.001^{* *}$ \\
\hline Delayed memory & $86.54 \pm 12.72$ & $80.44 \pm 14.63$ & 11.243 & $0.001^{* *}$ & 19.15 & $<0.001^{* *}$ \\
\hline RBANS total score & \multicolumn{7}{|l|}{} & & & \\
\hline Lipoprotein levels & $5.01 \pm 1.01$ & $4.85 \pm 0.77$ & 1.242 & 0.266 & 3.821 & $0.002^{* *}$ \\
\hline CHO (mmol/L) & $1.62 \pm 0.91$ & $1.57 \pm 0.88$ & 0.173 & 0.678 & 15.649 & $<0.001^{* *}$ \\
\hline TG (mmol/L) & $1.48 \pm 0.35$ & $1.54 \pm 0.32$ & 1.192 & 0.276 & 7.606 & $<0.001^{* *}$ \\
\hline HDL (mmol/L) & $2.81 \pm 0.90$ & $2.66 \pm 0.61$ & 1.680 & 0.196 & 3.654 & $0.003^{* *}$ \\
\hline LDL (mmol/L) &
\end{tabular}

Table 2. Cognitive performance and lipoprotein levels were significantly higher in the LD group than HD group after adjusting for the confounding factors. $L D$ low depressive, $H D$ high depressive. Adjusted F, $p$ values were controlled for age, gender, smoking and education years; ${ }^{*} p<0.05,{ }^{* *} p<0.01$.

Network estimation, centrality, network accuracy and network stability. A network of 11 nodes representing depressive state (SDS total score), lipoprotein levels (CHO, HDL, LDL and TG) and cognitive performance (immediate memory, visuospatial/constructional, language, attention, delayed memory, and RBANS total score) was constructed. As seen in Fig. 1, the community detection function identified three major communities, with depressive state (SDS total score) in red, lipoprotein indices (CHO, TG, LDL and HDL) in green, and cognitive performance (RBANS scores) in blue. Edges linking nodes within each community were the strongest, suggesting robust measurement validity. The nodes R_T (standing for "RBANS total score") and TG (triglycerides level) had the largest number of edges, and were situated in the center of the cognitive and serum lipoprotein communities respectively. In general, the node SDS had a prominent negative link with the cognitive community, suggesting adverse effect of depression on adult cognitive performance; whereas edges from the serum lipid community had both positive and negative connections with the other two communities. Firstly, LDL, CHO and TG levels were all negatively connected with the cognitive performance community. Secondly, TG level had a similar negative link to the SDS node, while CHO and LDL levels were irrelevant to depressive state after controlling for all other nodes. Only HDL level was positively connected to both self-rated depressive state and cognitive performance. Intriguingly, TG and CHO levels were positively linked as one "unit" or "axis". Within the lipid community, LDL and HDL each had one positive and one negative edge with this axis. LDL exhibited a strong positive connection to $\mathrm{CHO}$ and a weaker negative connection to TG. Inversely, HDL had a strong negative connection to TG and an equally strong but positive edge with $\mathrm{CHO}$.

Network inference measures showed that the SDS node had the highest standardized strength centrality in the network, followed by R_T, both of which also had the highest closeness estimate in the network, suggesting that they had the shortest path connecting them to other nodes. CS coefficients were 0.594 for strength, 0.516 for closeness, and below 0.25 for betweenness. Since the main indicator of stability was the CS coefficient of strength, which was above 0.5 , it was safe to assume that the current model was stable. The EI was at the maximum obtainable value of $0.75^{31}$. Additionally, results of the bootstrapped difference test, the edge weight accuracy test, and the edge weight difference test can be found in Supplementary Materials.

Correlations between lipoprotein profile, cognitive performance and depressive symptom. Table 3 shows that for the LD group $(n=379)$, CHO, TG and LDL all had significant negative correlations with cognitive scores. Specifically, scores on the memory related sub-scales (i.e. "Immediate Memory" and "Delayed Memory") were inversely correlated with CHO and LDL levels, while "Attention" was negatively correlated with TG level. Of the lipid profile indices, the correlation of HDL with cognitive performance was not significant. For the HD group $(\mathrm{n}=59)$, TG had a moderate to strong correlation with score on the "Attention" sub-scale of the RBANS ( $r=-0.317, p<0.05)$, while all other lipoprotein levels' correlation indices were not statistically significant.

\section{Discussion}

The present study examined the relationships among serum lipoprotein profile, cognitive impairment and selfrated depression using network analysis. The constructed network demonstrated the negative correlations of serum LDL, CHO and TG levels with cognitive performance. Moreover, TG level was negatively linked to depressive state, while HDL level was positively connected to both self-rated depressive state and cognitive performance. 


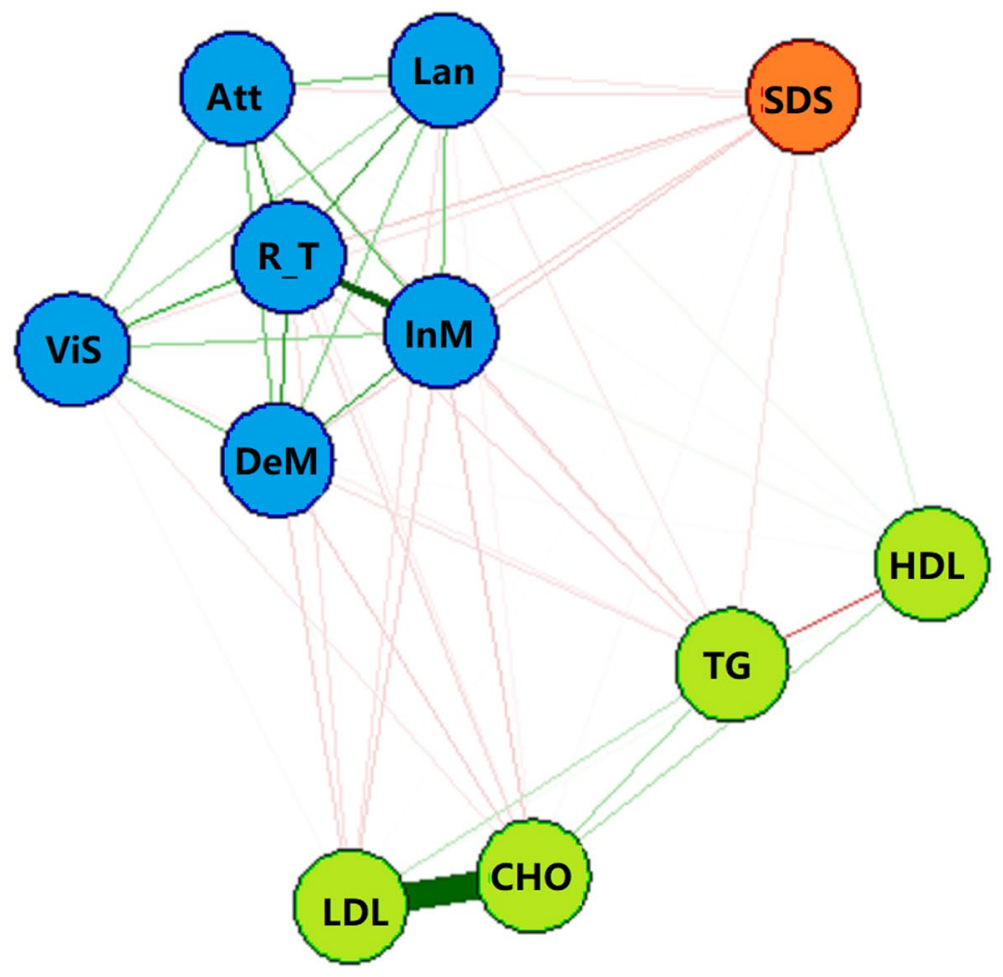

- SDS total score

- Lipoproteins

- RBANS scores

Figure 1. Network estimation of the whole study sample $(\mathrm{N}=438)$. Notes: Red edges indicate negative correlations, green edges indicate positive correlations, with thicker ones representing stronger partial correlations. Att Attention sub-scale of the RBANS, InM Immediate Memory sub-scale of the RBANS, ViS Visual spatial/Constructional sub-scale of the RBANS, Lan Language sub-scale of the RBANS, DeM Delayed Memory sub-scale of the RBANS, $R \_T$ RBANS total score, $C H O$ total cholesterol, $H D L$ high-density lipoprotein cholesterol, $L D L$ low-density lipoprotein cholesterol, TG triglycerides, SDS total score of the Self-Rating Depressive Scale.

\begin{tabular}{|l|l|l|l|l|l|l|r|r|r|}
\hline & \multicolumn{2}{|l}{ LD $(\mathbf{n}=\mathbf{3 7 9})$} & \multicolumn{2}{l|}{ HD $(\mathbf{n}=59)$} \\
\cline { 2 - 10 } & CHO & TG & HDL & LDL & CHO & TG & HDL & LDL \\
\hline Immediate memory & $-0.153^{* *}$ & -0.076 & 0.026 & $-0.140^{* *}$ & -0.082 & -0.121 & 0.059 & 0.001 \\
\hline Visual spatial/constructional & -0.059 & -0.048 & 0.018 & -0.017 & -0.168 & -0.021 & -0.049 & -0.018 \\
\hline Language & -0.014 & -0.034 & 0.022 & -0.081 & -0.178 & -0.185 & 0.143 & -0.137 \\
\hline Attention & -0.052 & $-0.103^{*}$ & 0.007 & -0.032 & -0.026 & $-0.317^{*}$ & 0.064 & 0.102 \\
\hline Delayed memory & $-0.159^{* *}$ & -0.087 & 0.009 & $-0.162^{* *}$ & 0.017 & -0.073 & -0.045 & 0.091 \\
\hline RBANS total Score & $-0.113^{*}$ & -0.093 & 0.027 & $-0.114^{*}$ & -0.089 & -0.190 & 0.062 & 0.024 \\
\hline
\end{tabular}

Table 3. Correlations between lipoprotein levels, TG, and cognitive performance across the LD and HD groups. Partial correlations, controlling for age, gender, smoking and education years, 2 -tailed, ${ }^{\star} p<0.05$, ${ }^{* *} p<0.01$.

Group comparison revealed that the HD group exhibited lower levels of serum LDL, CHO, and TG, higher level of serum HDL, and performed significantly worse in cognitive tests compared with the LD group.

Prior research suggests that cognitive impairment may be a core feature of self-rated depression ${ }^{15}$. In general, we found that self-rated depression had a significant negative impact on cognitive performance. Lipoprotein profile also differed significantly between the HD and LD groups after controlling for age, sex, education level and smoking status. Intriguingly, of the four serum lipid indices, only HDL displayed a positive correlation with cognitive function, and this correlation was significantly weakened in the HD group that exhibited higher HDL level, as well as lower CHO, TG and LDL levels (see Fig. 1 and Table 2). This result supports the negative correlation between TG level and cognitive impairment in major depressive disorder ${ }^{15}$. However, the negative relationship between high lipoprotein levels and cognitive performance did not appear in the correlation matrix of the HD group, which may be due to our small sample size $(n=59)$, or a genuine group-wise discrepancy in serum lipid profile.

Notwithstanding a well-recognized association between lipoprotein and depressive symptoms, the specific roles of CHO, TG, HDL and LDL are not clearly known. Our results are mostly consistent with the previously proposed "unfavourable serum lipid profile" featuring higher HDL, lower LDL and $\mathrm{CHO}^{24}$. Previous findings 
suggest that depressive symptoms are associated with lower LDL level in adult women ${ }^{18}$ and men $^{24}$, which are supported by our network analysis. However, contrary to studies that reported significant correlations of higher TG and CHO levels with depressive symptoms ${ }^{15,32}$, our network found that only HDL showed a positive association with self-rated depressive state.

A critical but unresolved issue therefore arises regarding whether HDL is protective against or contributes to depressive symptoms. We note that our finding of higher HDL level in the HD group contradicts with the results from most studies ${ }^{27,32}$. This inconsistency may reflect the complexity of HDL's relationship with depression, although variations in methodology (different self-report scales, covariates or confounders considered) along with demographics and ethnicity of the sample studied, may also account for the discrepancy. Another reason for the inconsistent results might lie in the nature of the depressive spectrum. In this study, group differences in participants with higher SDS scores (above 50 points) and lower scores (below 50 points) were compared, while some other studies investigated the change of HDL between patients with clinically diagnosed major depressive disorder and healthy controls. For example, results from a larger sample in the Netherlands Study of Depression and Anxiety (NESDA) indicated lower HDL level in 761 participants currently with major depressive disorder than 629 healthy controls $(p=0.007)^{32}$. However, such significance disappeared after controlling for lifestylerelated factors such as $\mathrm{BMI}^{32}$. Therefore, it is important to recognize that adverse lifestyle behaviors (e.g. lower dietary fibre intake and smoking) may underlie serum lipid levels which consequently affect cognitive and mood symptoms ${ }^{33}$. Consistent with our hypothesis, major demographic and lifestyle confounding factors (i.e. age, sex, education level and smoking status) exhibited a significant effect on serum lipid levels, cognition, depressive status and their associations.

This study has several limitations. Firstly, despite the present findings suggesting a correlational pattern between self-reported depression and lipoproteins, this study did not empirically test the directionality of effect between depressive symptoms and serum lipid levels ${ }^{24}$. Secondly, due to difficulties in data collection, the comparison between depressive and non-depressive individuals in our samples was not matched for age and intelligence quotient (IQ), which might have biased our results. Further research would benefit from a larger and more demographically-balanced sample. Also, exclusion of participants with diagnosis of major depressive disorder is a major limitation for us to address the association between depressive symptoms, cognition and lipoprotein profile. Thus, future study should recruit participants with established major depressive disorder to examine whether a similar network pattern would be shown. Finally, our sample size was relatively small $(n=438)$ for network analysis, which limited our network's stability. Future studies should investigate the relationship between increased HDL level and cognitive impairment in people with major depressive disorder and examine how this contributes to the core symptoms of major depressive disorder.

In summary, our results suggest that there may be distinct correlations between serum lipoprotein levels, cognitive performance and self-rated depressive status. The use of network analysis has added to the current knowledge in this field by illustrating the relationships between specific lipoprotein levels, cognitive function and self-rated depression after controlling for all other factors in the conceptual network. Serum lipoprotein levels may play a putative role in cognitive impairment in people with self-rated depression.

\section{Methods}

Participants. Participants were recruited from the annual physical examination event of the Wenzhou Kangning Hospital. A total of 575 people attended the event, and 512 people agreed to take part in the study upon invitation. Seventy-four participants were excluded due to physical health problems, taking regular medications and returning incomplete checklists. The final sample consisted of 438 adults, with 312 females and 126 males. The following inclusion criteria were applied: (1) staff of the hospital; (2) 18-70 years of age; (3) no history of mental illness that was diagnosed in the course of the physical and psychological examinations of newly recruited staff; (4) no history of alcohol and drug dependence; and (5) good past physical health. The study protocol was approved by the Ethics Committee of the Wenzhou Kangning Hospital, and all procedures were carried out in accordance with the approved guidelines and regulations. Written informed consent was obtained from each participant.

Measurements. Cognitive performance was assessed using the RBANS ${ }^{34}$. The RBANS consists of 12 subtests that can be generalized into five sub-scale scores and a total score. The five sub-scales are (1) Immediate Memory (consisting of list learning and story memory tests); (2) Visuospatial/Constructional (consisting of figure copying and line orientation tests); (3) Language (consisting of picture naming and semantic fluency tests); (4) Attention (consisting of digit span and coding tests); and (5) Delayed Memory (consisting of list recall, story recall, figure recall and list recognition tests). Participants had to complete all tests to obtain a total cognitive score. The present study adopted the Chinese version of the RBANS that has been shown to have good test-retest reliability and validity in a healthy Chinese population ${ }^{35}$.

The SDS is a 20 -item checklist which assesses the depressive state of participants ${ }^{36}$. The original SDS had a sensitivity of $97 \%$, a specificity of $63 \%$, and an $82 \%$ correct classification rate in distinguishing between depressed and non-depressed individuals. The responses were rated from 0 to 3 and summed to obtain a total score, which was then transformed into a standard score ${ }^{37}$. Based on a morbidity cutoff index from previous research ${ }^{38}$, participants who had a SDS standard score below 50 points were classified into the LD group, and those with a score above 50 were classified into the HD group in the present study.

Serum lipoprotein levels. The overnight fasting blood sample was collected from all participants by venipuncture, between 7:00 and 9:00 am. The sample of approximately $5 \mathrm{ml}$ venous blood was allowed to clot at room temperature, and then centrifuged at $3500 \mathrm{rpm}$ for four minutes. After collecting the serum, the sample 
was stored at $-80^{\circ} \mathrm{C}$ until it was thawed for assay. CHO, HDL, LDL and TG levels were measured by enzymatic colourimetry with a HITACHI 7180 automatic biochemistry analyzer (Hitachi High-Technologies Corporation, Japan) and commercially available kits (Medical System Biotechnology, Ningbo, China).

Network estimation and statistical analysis. Network analysis is a new tool that visualizes partially controlled relational links among conceptualized factors ${ }^{39}$. In this study, we used the R software (version 3.5.0, available at https://cran.r-project.org/) to construct a partial correlation network encompassing data from all participants $(\mathrm{n}=438)$. A total of 11 nodes representing: (1) lipid profile encompassing CHO, HDL, LDL, and TG levels; (2) a self-rated depressive state indexed by the SDS total score; and (3) cognitive performance level indexed by the total score and scores on the five sub-scales of the RBANS, were included to explore the relationships among lipoprotein levels, self-rated depressive state and cognitive performance of the participants. Two measures were applied to estimate network inference: Strength Centrality and Expected Influence (EI). Strength Centrality measures the number of direct connections of one node in the whole network, which connotes the importance of a particular node. One step EI is the sum of all edges of a node, while two-step EI measures the accuracy and stability of the constructed network. The bootstrapped difference test, the edge weight accuracy test, and the edge weight difference test were applied to all node centrality indices. The stability of the centrality estimates was also quantified by the correlation-stability-coefficient ${ }^{40}$.

To analyze the differences in lipoprotein levels and cognitive performance between the LD and HD groups, multivariate ANOVA was conducted while controlling for four major confounding factors (i.e. age, sex, education level and smoking status) using SPSS 16.0 (https://www.ibm.com/analytics/spss-statistics-software). Due to the small number of participants in the HD group $(n=59)$, we applied an additional partial correlation analysis to investigate the between-group differences in the influence of serum lipid levels on cognitive performance.

Received: 10 June 2020; Accepted: 17 November 2020

Published online: 26 November 2020

\section{References}

1. Bauer, L. et al. A brief neuropsychological battery for use in the chronic heart failure population. Eur. J. Cardiovasc. Nurs. J. 11, 223-230 (2012).

2. Rock, P. L., Roiser, J. P., Riedel, W. J. \& Blackwell, A. D. Cognitive impairment in depression: a systematic review and meta-analysis. Psychol. Med. 44, 2029-2040 (2014).

3. Bates, K. A. et al. Serum high-density lipoprotein is associated with better cognitive function in a cross-sectional study of aging women. Int. J. Neurosci. 127, 243-252 (2017).

4. Chanti-Ketterl, M., Andel, R., Lerch, O., Laczo, J. \& Hort, J. Cholesterol and cognitive performance among community volunteers from the Czech Republic. Int. Psychogeriatr. 27, 2087-2095 (2015).

5. Choi, H. et al. Serum high-density lipoprotein cholesterol concentration and functional state: the Korean Urban Rural Elderly (KURE) Study. Arch. Gerontol. Geriatr. 71, 115-121 (2017).

6. Formiga, F. et al. Serum high-density lipoprotein cholesterol levels correlate well with functional but not with cognitive status in 85-year-old subjects. J. Nutr. Health Aging 16, 449-453 (2012).

7. Henderson, V. W., Guthrie, J. R. \& Dennerstein, L. Serum lipids and memory in a population based cohort of middle age women. J. Neurol. Neurosurg. Psychiatry 74, 1530-1535 (2003).

8. Corley, J., Starr, J. M. \& Deary, I. J. Serum cholesterol and cognitive functions: the Lothian Birth Cohort 1936. Int. Psychogeriatr. 27, 439-453 (2015).

9. van den Kommer, T. N., Dik, M. G., Comijs, H. C., Jonker, C. \& Deeg, D. J. Role of lipoproteins and inflammation in cognitive decline: do they interact?. Neurobiol. Aging 33(196), e191-e112 (2012).

10. Leritz, E. C., McGlinchey, R. E., Salat, D. H. \& Milberg, W. P. Elevated levels of serum cholesterol are associated with better performance on tasks of episodic memory. Metab. Brain Dis. 31, 465-473 (2016).

11. Crichton, G. E., Elias, M. F., Davey, A., Sullivan, K. J. \& Robbins, M. A. Higher HDL cholesterol is associated with better cognitive function: the Maine-Syracuse study. J. Int. Neuropsychol. Soc. JINS 20, 961-970 (2014).

12. Huang, C. Q. et al. Association of cognitive impairment with serum lipid/lipoprotein among Chinese nonagenarians and centenarians. Dement. Geriatr. Cogn. Disord. 27, 111-116 (2009).

13. Packard, C. J. et al. Association between apolipoprotein E4 and cognitive decline in elderly adults. J. Am. Geriatr. Soc. 55, 1777-1785 (2007).

14. Granholm, A. C. et al. Effects of a saturated fat and high cholesterol diet on memory and hippocampal morphology in the middleaged rat. J. Alzheimer's Dis. JAD 14, 133-145 (2008).

15. Shao, T. N. et al. Elevated triglyceride levels are associated with cognitive impairments among patients with major depressive disorder. Compr. Psychiatry 75, 103-109 (2017).

16. Aijanseppa, S. et al. Serum cholesterol and depressive symptoms in elderly Finnish men. Int. J. Geriatr. Psychiatry 17, 629-634 (2002).

17. Steegmans, P. H., Hoes, A. W., Bak, A. A., van der Does, E. \& Grobbee, D. E. Higher prevalence of depressive symptoms in middleaged men with low serum cholesterol levels. Psychosom. Med. 62, 205-211 (2000).

18. Fang, C. Y. et al. Depressive symptoms and serum lipid levels in young adult women. J. Behav. Med. 36, 143-152 (2013).

19. Beydoun, M. A. et al. Total serum cholesterol, atherogenic indices and their longitudinal association with depressive symptoms among US adults. Transl. Psychiatry 5, e518 (2015).

20. Ji-Rong, Y., Bi-Rong, D., Chang-Quan, H. \& Yan-Ling, Z. Depression and serum lipids and lipoprotein in Chinese nonagenarians and centenarians. J. Am. Geriatr. Soc. 57, 732-733 (2009).

21. Bots, S., Tijhuis, M., Giampaoli, S., Kromhout, D. \& Nissinen, A. Lifestyle- and diet-related factors in late-life depression-a 5-year follow-up of elderly European men: the FINE study. Int. J. Geriatr. Psychiatry 23, 478-484 (2008).

22. Olusi, S. O. \& Fido, A. A. Serum lipid concentrations in patients with major depressive disorder. Biol. Psychiatry 40, 1128-1131 (1996).

23. Rabe-Jablonska, J. \& Poprawska, I. Levels of serum total cholesterol and LDL-cholesterol in patients with major depression in acute period and remission. Med. Sci. Monit. 6, 539-547 (2000).

24. Igna, C. V., Julkunen, J., Vanhanen, H., Keskivaara, P. \& Verkasalo, M. Depressive symptoms and serum lipid fractions in middleaged men: physiologic and health behavior links. Psychosom. Med. 70, 960-966 (2008). 
25. Liang, Y. et al. Association between lipid profile and depressive symptoms among Chinese older people: mediation by cardiovascular diseases?. Int. J. Behav. Med. 21, 590-596 (2014).

26. Lehto, S. M. et al. Elevated depressive symptoms and compositional changes in LDL particles in middle-aged men. Eur. J. Epidemiol. 25, 403-409 (2010).

27. Pan, A. et al. Bidirectional association between depression and metabolic syndrome a systematic review and meta-analysis of epidemiological studies. Diabetes Care 35, 1171-1180 (2012).

28. Persons, J. E. \& Fiedorowicz, J. G. Depression and serum low-density lipoprotein: a systematic review and meta-analysis. J. Affect. Disord. 206, 55-67 (2016).

29. Chen, C. C., Lu, F. H., Wu, J. S. \& Chang, C. J. Correlation between serum lipid concentrations and psychological distress. Psychiatry Res. 102, 153-162 (2001).

30. Borsboom, D. \& Cramer, A. O. Network analysis: an integrative approach to the structure of psychopathology. Annu. Rev. Clin. Psychol. 9, 91-121 (2013).

31. Robinaugh, D. J., Millner, A. J. \& McNally, R. J. Identifying highly influential nodes in the complicated grief network. J. Abnorm. Psychol. 125, 747-757 (2016).

32. van Reedt Dortland, A. K. et al. Associations between serum lipids and major depressive disorder: results from the Netherlands Study of Depression and Anxiety (NESDA). J. Clin. Psychiatry 71, 729-736 (2010).

33. Gravely-Witte, S., Stewart, D. E., Suskin, N. \& Grace, S. L. The association among depressive symptoms, smoking status and antidepressant use in cardiac outpatients. J. Behav. Med. 32, 478-490 (2009).

34. Randolph, C., Tierney, M. C., Mohr, E. \& Chase, T. N. The repeatable battery for the assessment of neuropsychological status (RBANS): preliminary clinical validity. J. Clin. Exp. Neuropsychol. 20, 310-319 (1998).

35. Zhang, B. H. et al. Repeatable battery for the assessment of neuropsychological status as a screening test in Chinese: reliability and validity. Chin. Ment. Health J. 22, 865-869 (2009).

36. Zung, W. W., Magruder-Habib, K., Velez, R. \& Alling, W. The comorbidity of anxiety and depression in general medical patients: a longitudinal study. The Journal of clinical psychiatry 51(Suppl), 77-80 (1990) (discussion 81).

37. Liu, X. C. et al. Factor analysis of self-rating depression scale (SDS). Clin. Psychol. 3, 151-154 (1994).

38. Gao, Y., Wang, W., Huang, D. \& Liu, H. Analysis of anxiety and depression status and influencing factors among parturient pregnant women. Anhui Med. J. 35, 493-496 (2014).

39. Borsboom, D. Psychometric perspectives on diagnostic systems. J. Clin. Psychol. 64, 1089-1108 (2008).

40. Epskamp, S., Borsboom, D. \& Fried, E. I. Estimating psychological networks and their accuracy: a tutorial paper. Behav. Res. Methods 50, 195-212 (2018).

\section{Acknowledgements}

This study was supported by the National Natural Science Foundation of China (81771439), Jiangsu Provincial Key Research and Development Program (BE2020661), Jiangsu Provincial Health Commission Science Research Program (H2019056 and LGY2018010), Jiangsu Provincial High-level Health Talent Project (QNRC2016228), Jiangsu Provincial Six Talent Peaks Project (WSN-165), Suzhou Municipal Health Commission Program (LCZX201820 and Gwzx201801), Suzhou Municipal Sci-Tech Bureau Program (SZS201722, SYS2019113 and SS2019009), and CAS Key Laboratory of Mental Health, Institute of Psychology (KLMH2019K03).

\section{Author contributions}

Q.F.J., H.X.Y., N.N.Z. and L.H. contributed to the overall design of this study, conducted the statistical analysis, and wrote the first draft of manuscript. Q.F.J., H.X.Y., N.N.Z., X.Y.Y., Z.H.Z., Y.Y., X.L.Y. and L.H. recruited the subjects, performed the clinical assessment, and collected the serum sample. Y.W. and E.F. C. C. interpreted the findings and commented the drafts critically. R.C.K.C generated the idea of the study, interpreted the findings and commented the drafts critically. L.H. was responsible for the protocol and manuscript, and provided the funding for this study. Each author approved the final manuscript.

\section{Competing interests}

The authors declare no competing interests.

\section{Additional information}

Supplementary information is available for this paper at https://doi.org/10.1038/s41598-020-77782-9.

Correspondence and requests for materials should be addressed to R.C.K.C. or L.H.

Reprints and permissions information is available at www.nature.com/reprints.

Publisher's note Springer Nature remains neutral with regard to jurisdictional claims in published maps and institutional affiliations.

(c) (i) Open Access This article is licensed under a Creative Commons Attribution 4.0 International cc) License, which permits use, sharing, adaptation, distribution and reproduction in any medium or format, as long as you give appropriate credit to the original author(s) and the source, provide a link to the Creative Commons licence, and indicate if changes were made. The images or other third party material in this article are included in the article's Creative Commons licence, unless indicated otherwise in a credit line to the material. If material is not included in the article's Creative Commons licence and your intended use is not permitted by statutory regulation or exceeds the permitted use, you will need to obtain permission directly from the copyright holder. To view a copy of this licence, visit http://creativecommons.org/licenses/by/4.0/.

(c) The Author(s) 2020 